\title{
Design and Research of Network Dynamic Sports Service Industry Management Model
}

\author{
Guo sheng \\ P. E. College, Beihua University \\ Jilin City, Jilin Province, China
}

Keywords: Mean server manager, Sports management service, Dynamic management, JMX.

\begin{abstract}
At present, Information System is facing a big challenge: How to effectively manage legacy management system (EMS, NMS), simultaneously ,also can manage new services which are joining at any moment. in this paper, we first analyze the technology background and architecture of JMX. then design and implement a JMX-based dynamic network sports management service model, which can effective and convenient for managing legacy system, but also can seamless add new service to the system. Thus, this system provide a perfect resolution for the challenge, it will have a good future for implementation.
\end{abstract}

\section{Introduction}

The complexity of IT network is increasing dramatically in modern times. So how to manage new network services while managing original management systems (EMS, NMS) effectively has become a difficult challenge for traditional NSM (Network Management System) software system structure. In this new environment, there is an urgent need for a flexible management solution which can meet the need of growing sports service industry management and can be easily implemented. It should also have good expansibility and can be compatible with original system. Expanding the network dynamic business is needed to deploy the mechanism of sports service industry management.

In the field of network management, latest technology and matched JMX development tools build a brand new system model for network dynamic service. It mainly use JMX architecture to package various network services into Dynamic Management Bean of JMX, release their control interface to remote administrator through agent, and new developed protocol adapter can also be quickly integrated into system at the same time. Then the administrator may write any management protocol interface to manage all services. Thus, a complete solution is formed for network service management.

\section{Overview of JMX architecture}

JMX (Java Management Extensions) is a framework to implant management function for application, device, system etc. It is committed to solve distributed system management issues. It can span a range of heterogeneous operating system platforms, system architecture and network transmission protocol, which can be used to flexibly develop seamless integration system, network application and service management application. Its value is to abstract managed resource service, provide a set of low-level base class, and under the premise of ensuring the integrity and consistency of most public management class, developers may extend it to meet the need of specific network management application. JMX architecture is composed of 4 layers shown in Figure 1. 


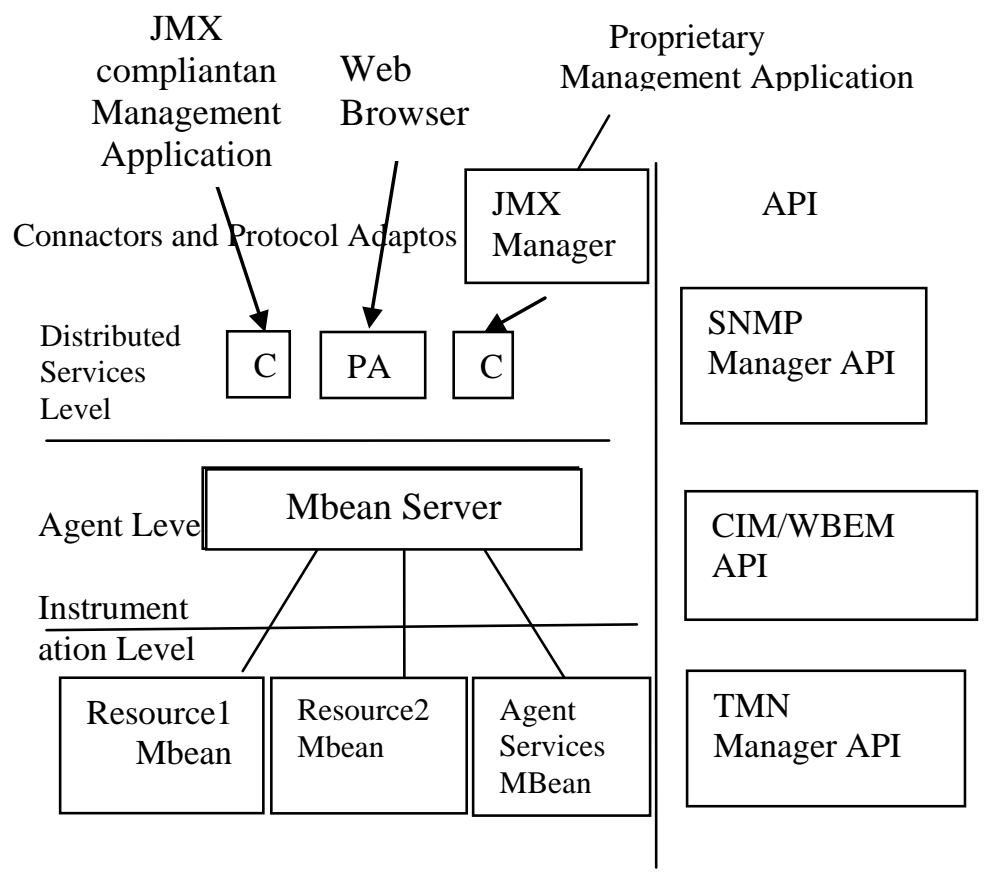

Figure 1 JMX system structure

Device layer. This layer mainly defines the information model of managed resource. The managed resource here is a very broad concept, which can be an application, service, or device. The layer also defines the notification mechanism as well as some auxiliary metadata class. MBean informs the information of state change to management procedure and Agent by JMX Notification Model, and the Agent get information of MBean from Metadata. JMX defines 4 types of MBean standard MBean, dynamic MBean, model MBean, and open MBean.

Agent layer. This layer defines various services and communication models. It runs on a Java virtual machine as management entity. It is active in management resource and administrator to manage resource directly, and makes these resources be controlled by remote management procedure. The Agent is usually composed of an MBean Server and several system services. Furthermore, it provides Adapter(s) or Connector(s) for outside access. The core of this layer is an MBean Server and all management components need to register it to be managed. A unique object name has to be provided to register a management component. Then management application identifies and operates management component with this object name. The management component registering on MBean Server does not communicate with remote application directly, but communicates with the connector by protocol adapter. And the adapter and connector must register on MBean Server in the form of management component to provide relevant service.

Distributed service layer. This layer defines management interface and component that operate the agent layer so the administrator can operate agent. It is mainly concerned about the details of how to access the Agent for remote user. It defines a series of interfaces and components that are used to access the Agent, including description of the Adapter and Connector, as well as application of connecting with the outside world through them, such as RMI management application, browserbased management control, etc.

Additional management protocol API. Defined API is mainly used to support the existing network management protocols, such as SNMP, TMN, CIM/WBEM, RMI/IIOP, etc. These API can help developers deploy their management platform and application and interact with management environment according to the most commonly used industrial standard, then this interaction process will be packaged in a JMX management resource, which makes new network management solution and existing basic structure into one. 


\section{Operating model of JMX}

Figure 2 describes the key operation of management software and managed application system under JMX architecture. This figure shows the application software deployed on J2EE Application Server, and application developer transforms the program resource needed to be managed into MBean. This MBean has to register on MBean Server in JMX facilities before it runs, and calls managed resource to complete management task in the process of its running. Management software has to access MBean through MBean Server. The MBean Server however uses a unique Object Name to identify and access registered MBean. Local management software may belong to the same virtual machine with the application software to access MBean Server without remote protocol. Remote management software can communicate with JMX agent through SN-MP adapter, HTTP adapter and various connecters.

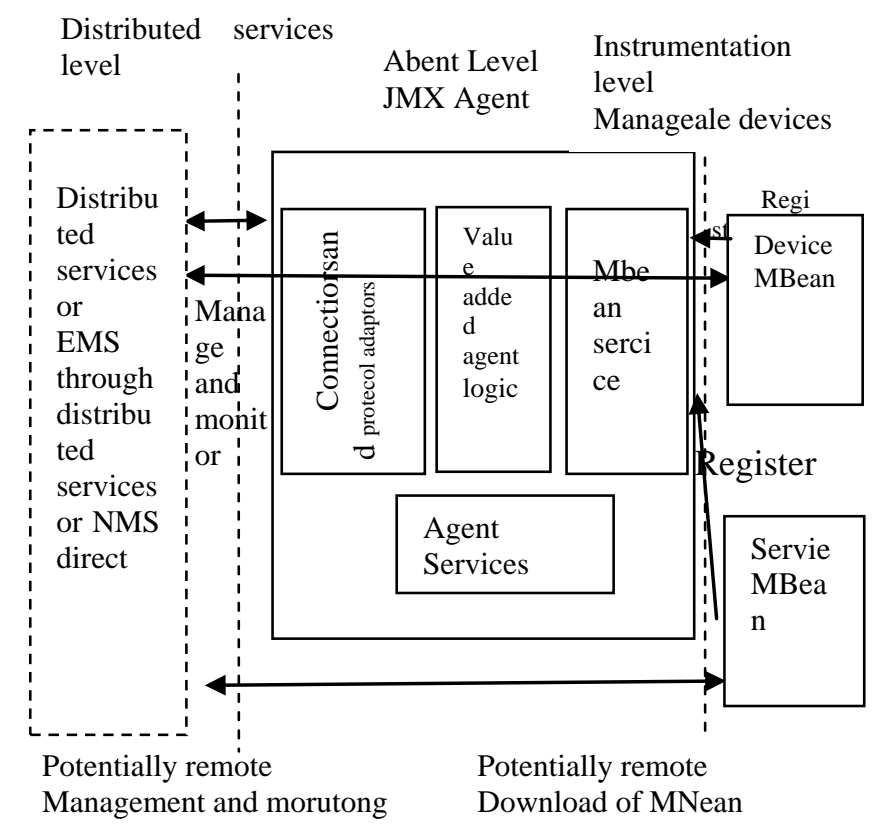

Figure 2 JMX operation model

\section{References}

[1] Dario GL, Aron SB, Ian MF. Enhancement of motor rehabilita-tion through the use of information technologies. Clinical Biome-chanics, 2006; 21(2) $: 8$

[2] Yoram B, Ariel M. Auditory feedback control for improvement of gait in patients with Multiple Sclerosis. Journal of the Neurological Sciences, 2007; 254(1-2) : 90

[3] Jeffrey MS, Carl WL, Louis P,et al.Discrete bandwidth visual feedback increases structure of output as compared to continuous visual feedback in isometric force control tasks. Clinical Biomechanics, 2006; 21(10) : 1042

[4] Han R, Ni CM. Effect of electromygraphic biofeedback on upper extremity function in patients with hemiplegia after stroke. Chin J Rehabil Theory Practice,2005;11(3)

[5] Liu YH, Zhang SZ, Ge RL. The clinical observation on the treat-ment of ankle disorder of paralysis with EMG feedback. Chinese Journal of Rehabilitation Medicine, 2005; 20(5) : 379 